March 13, 2006

Supporting Information for

\title{
Hybrid Density Functional Theory with Specific Reaction Parameter: Hydrogen Abstraction Reaction of Fluoromethane by the Hydroxyl Radical
}

\author{
Titus V. Albu* and Saravanan Swaminathan \\ Department of Chemistry, Box 5055, Tennessee Technological University, \\ Cookeville, Tennessee 38505
}

In this supporting information, we present:

- Additional dynamics results obtained on the investigated HDFT surfaces (Tables 1-S to 9-S)

- Dynamics results obtained when scaling the frequencies with a multiplicative factor (Tables 10-S to $12-\mathrm{S})$

- The parameters from the quadratic fit of the rate constant vs. $X$ (the HF exchange contribution) (Table 13-S)

- Optimized geometries, energies and frequencies for reactants, products, and saddle point for all HDFT methods examined

* Corresponding author e-mail: albu@tntech.edu 


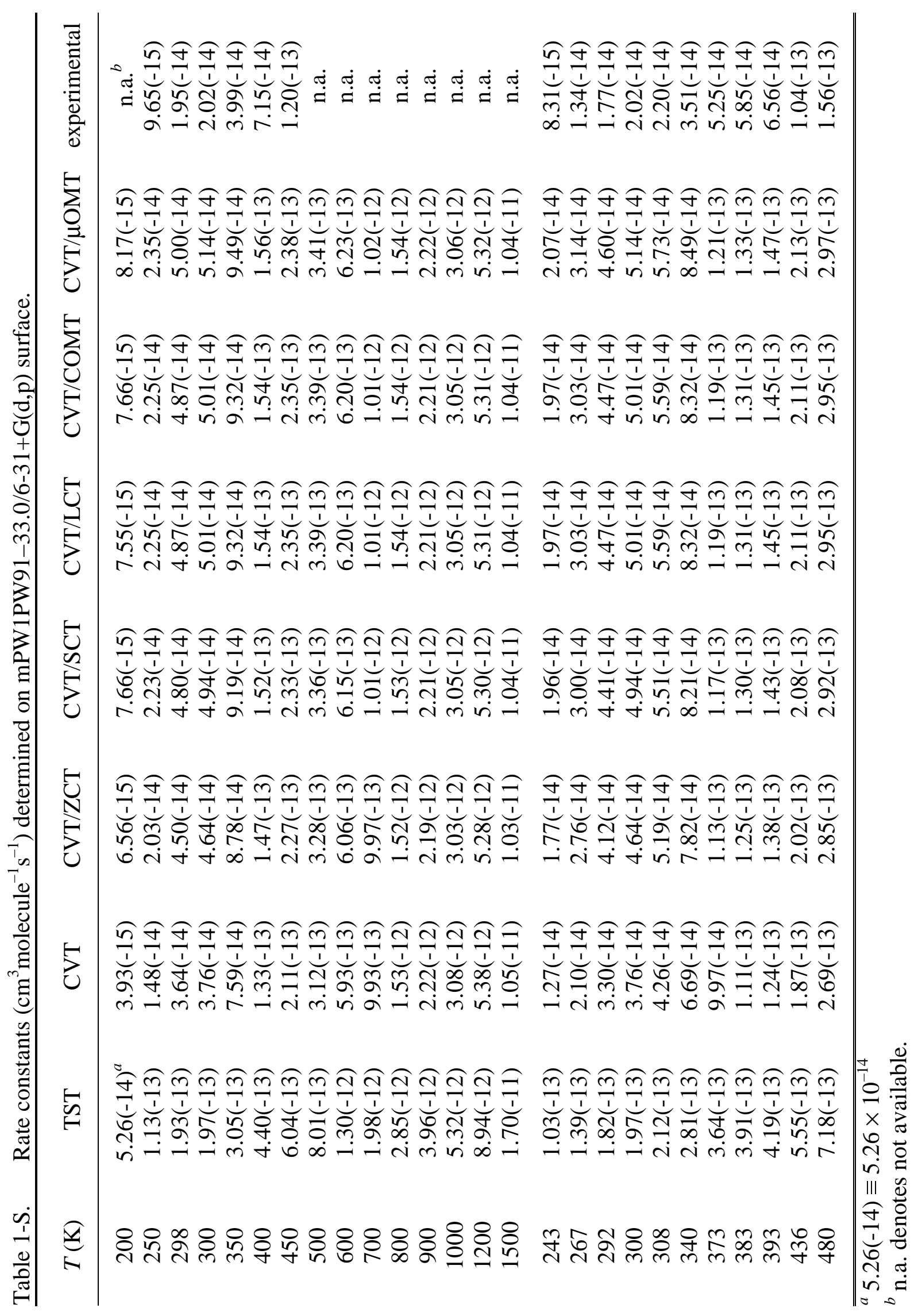




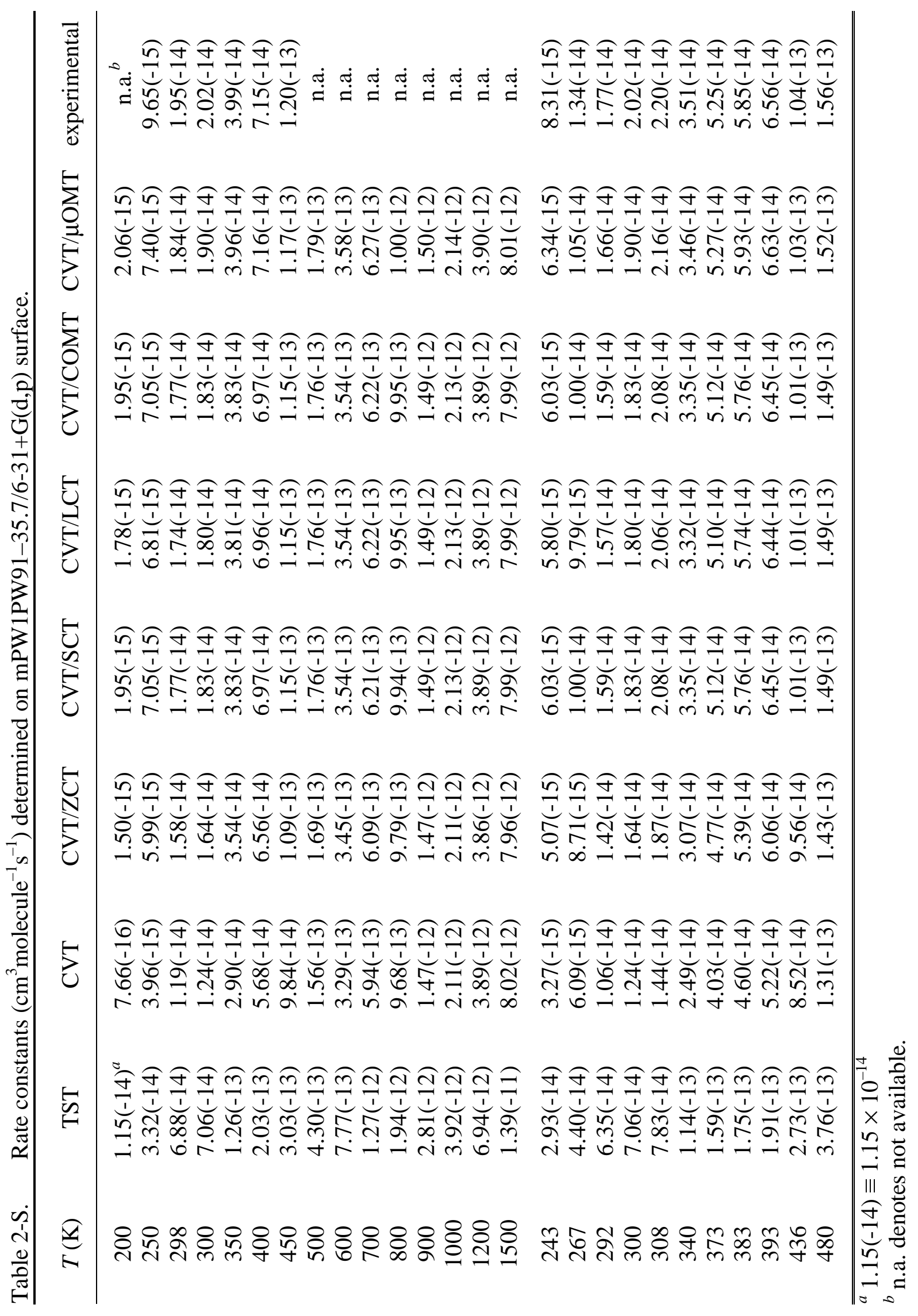




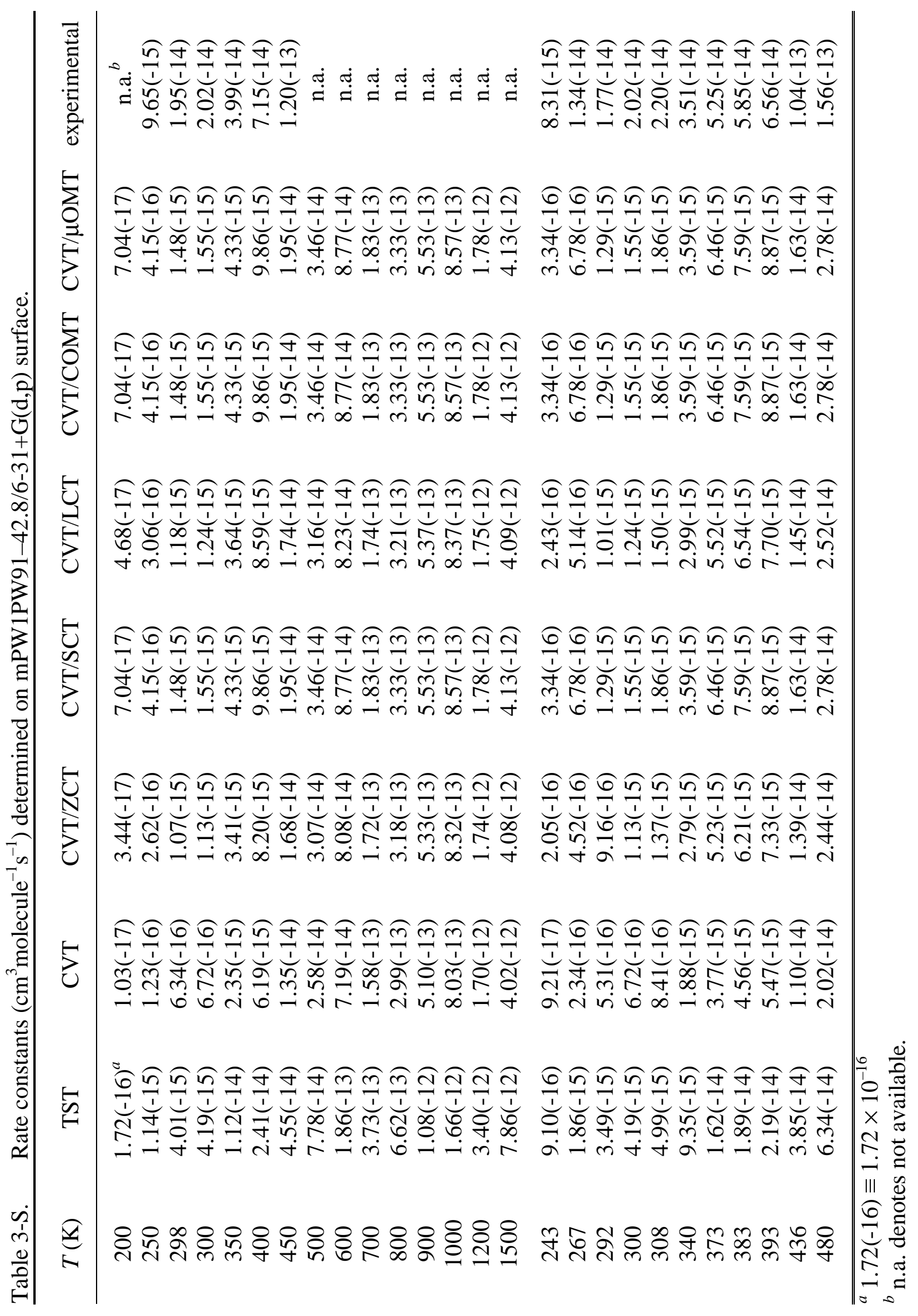




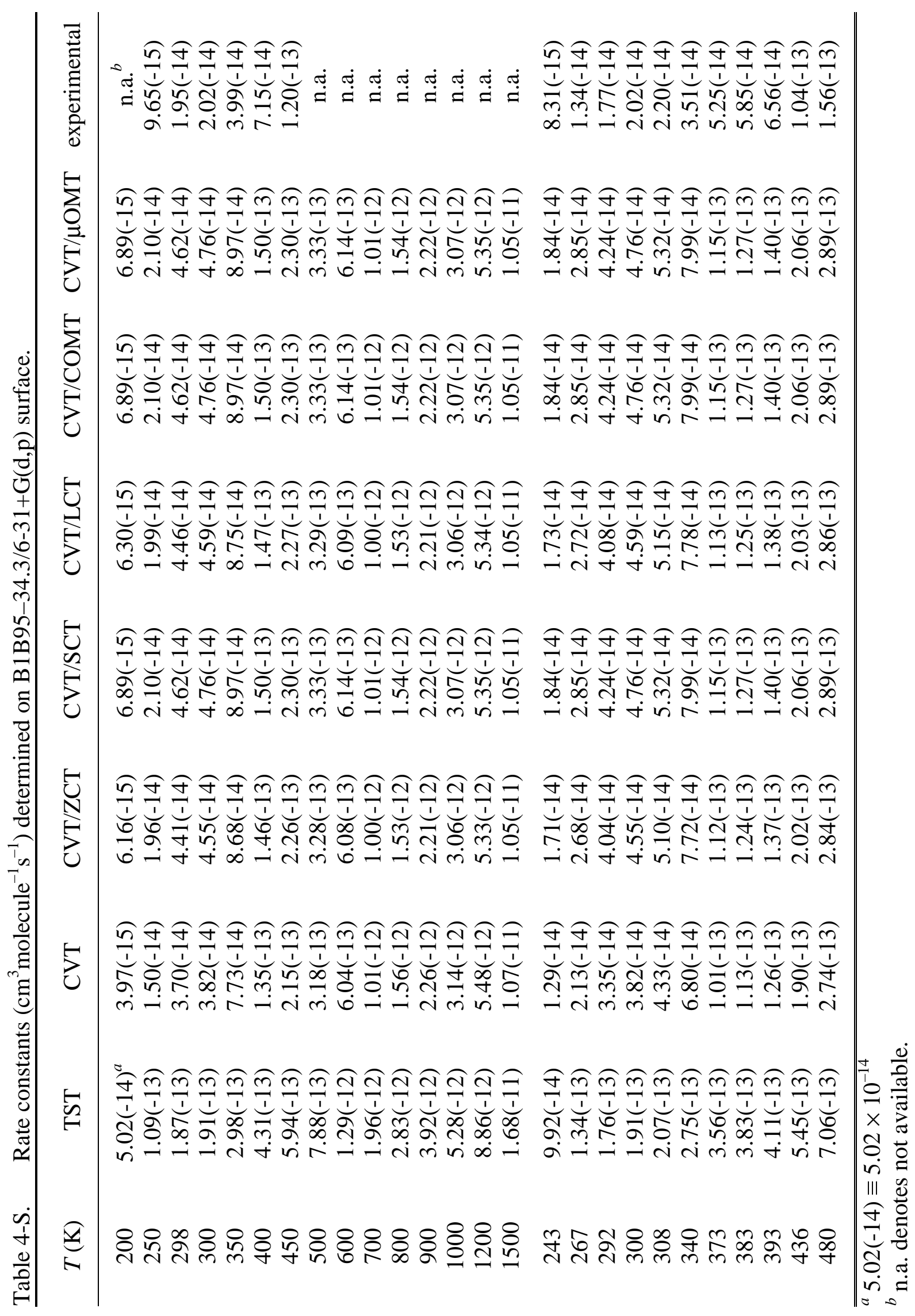




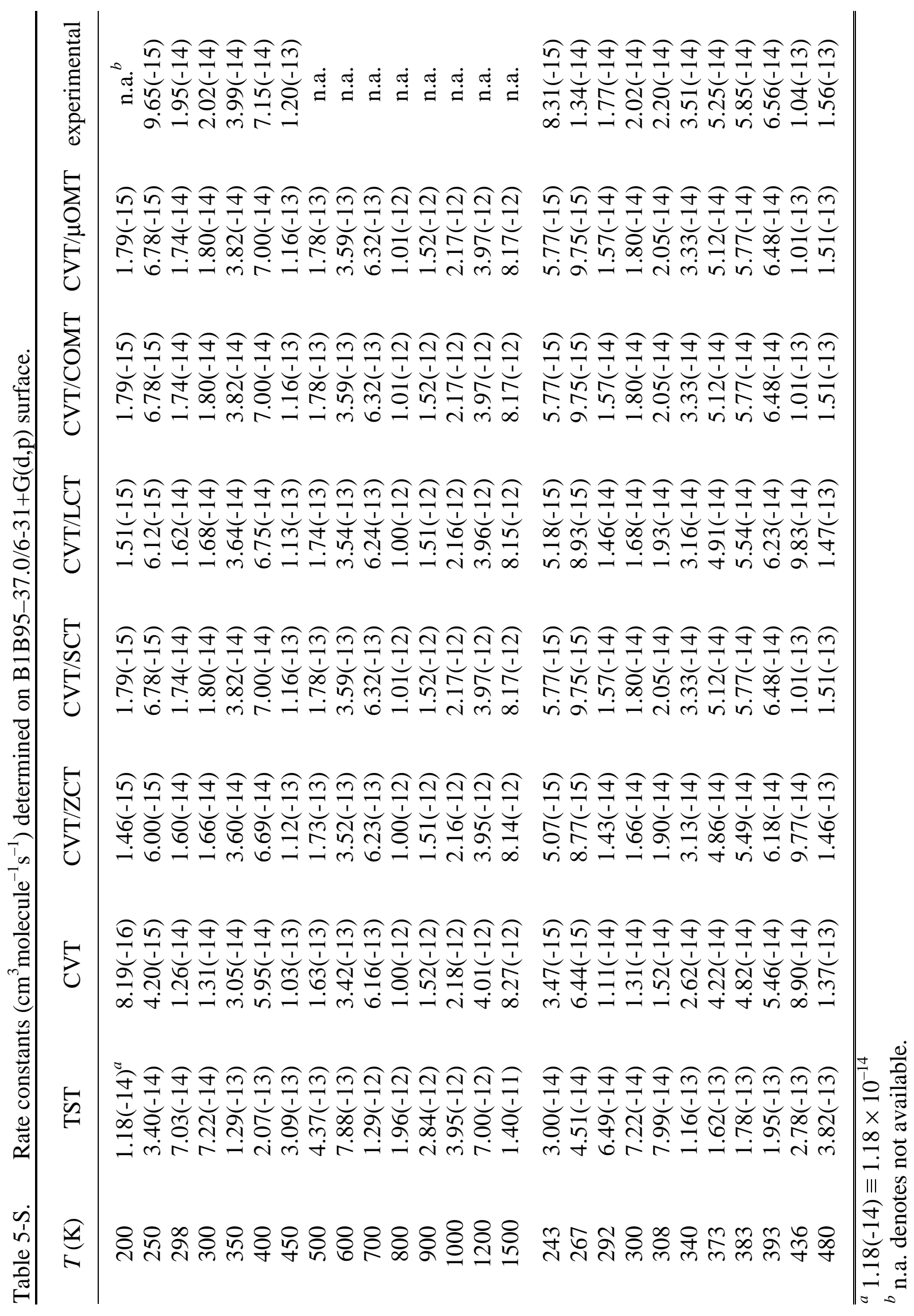




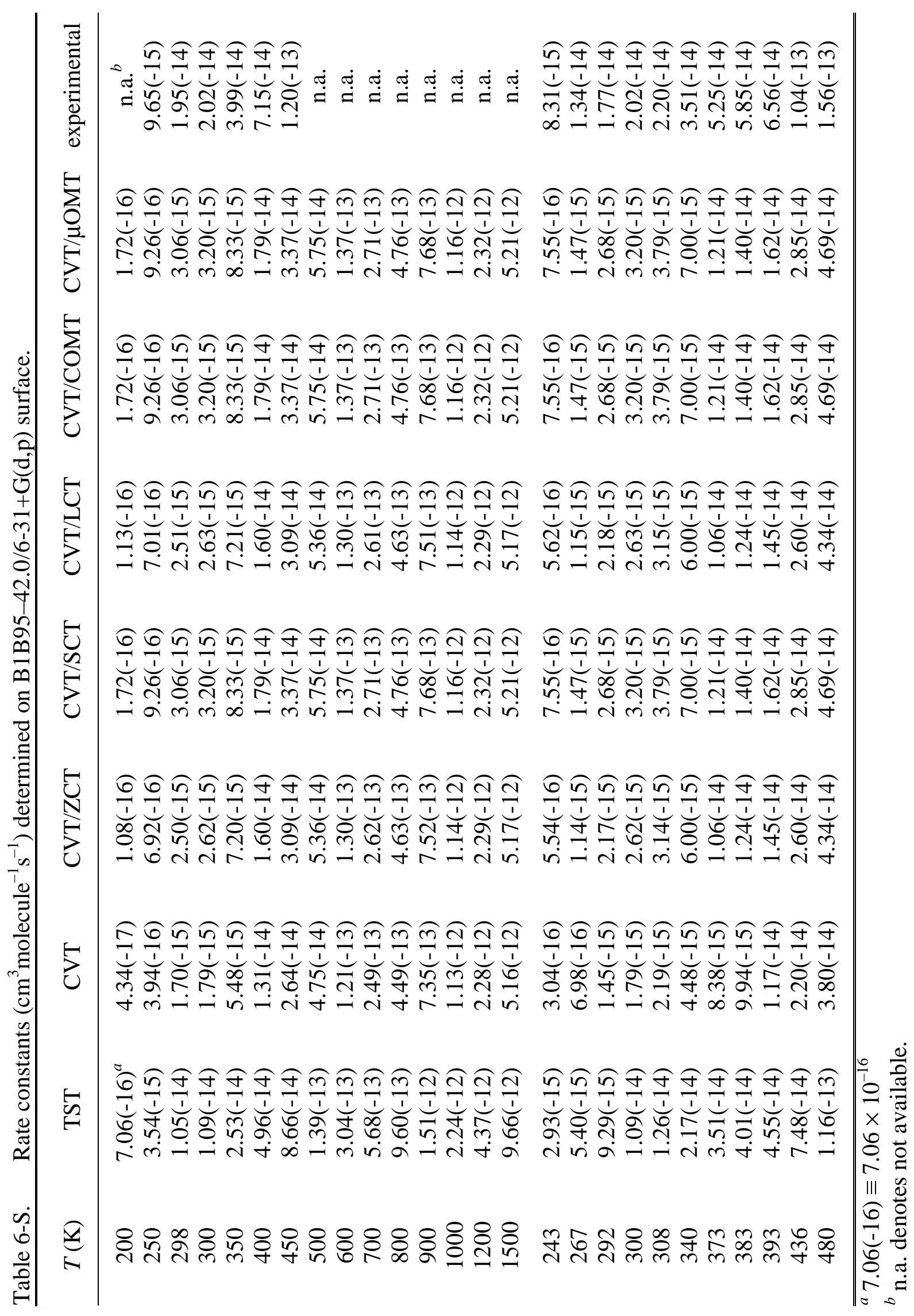




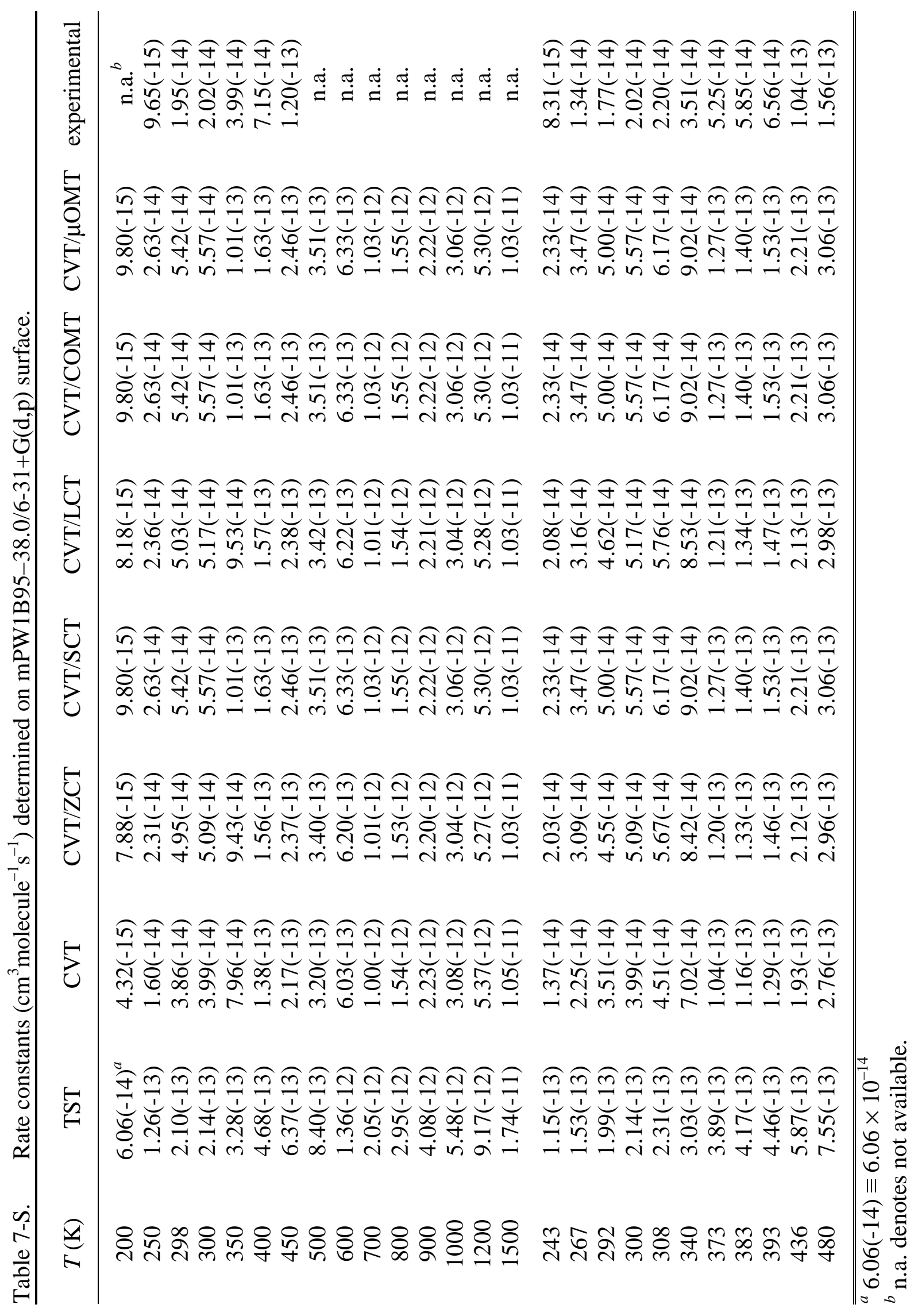




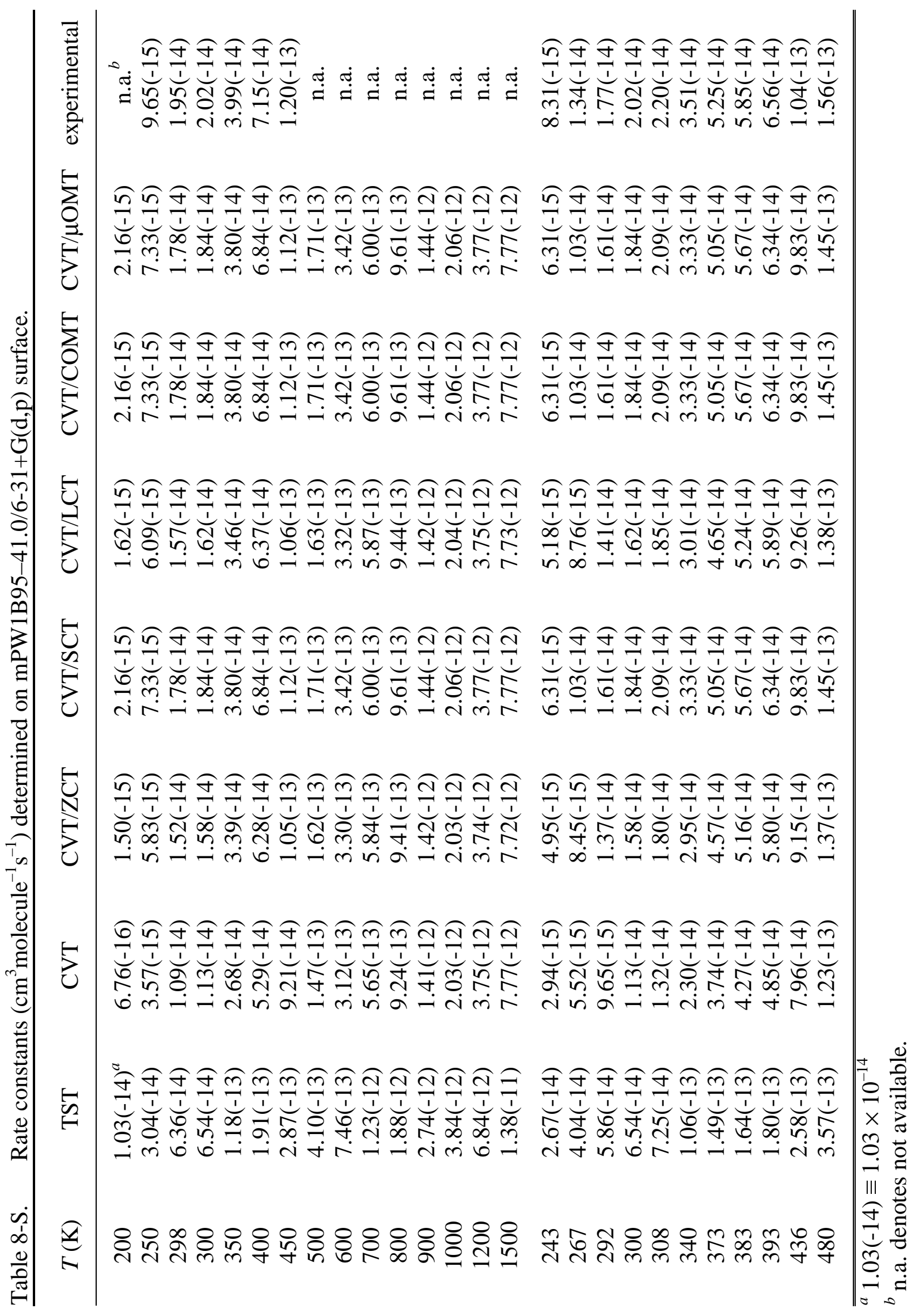




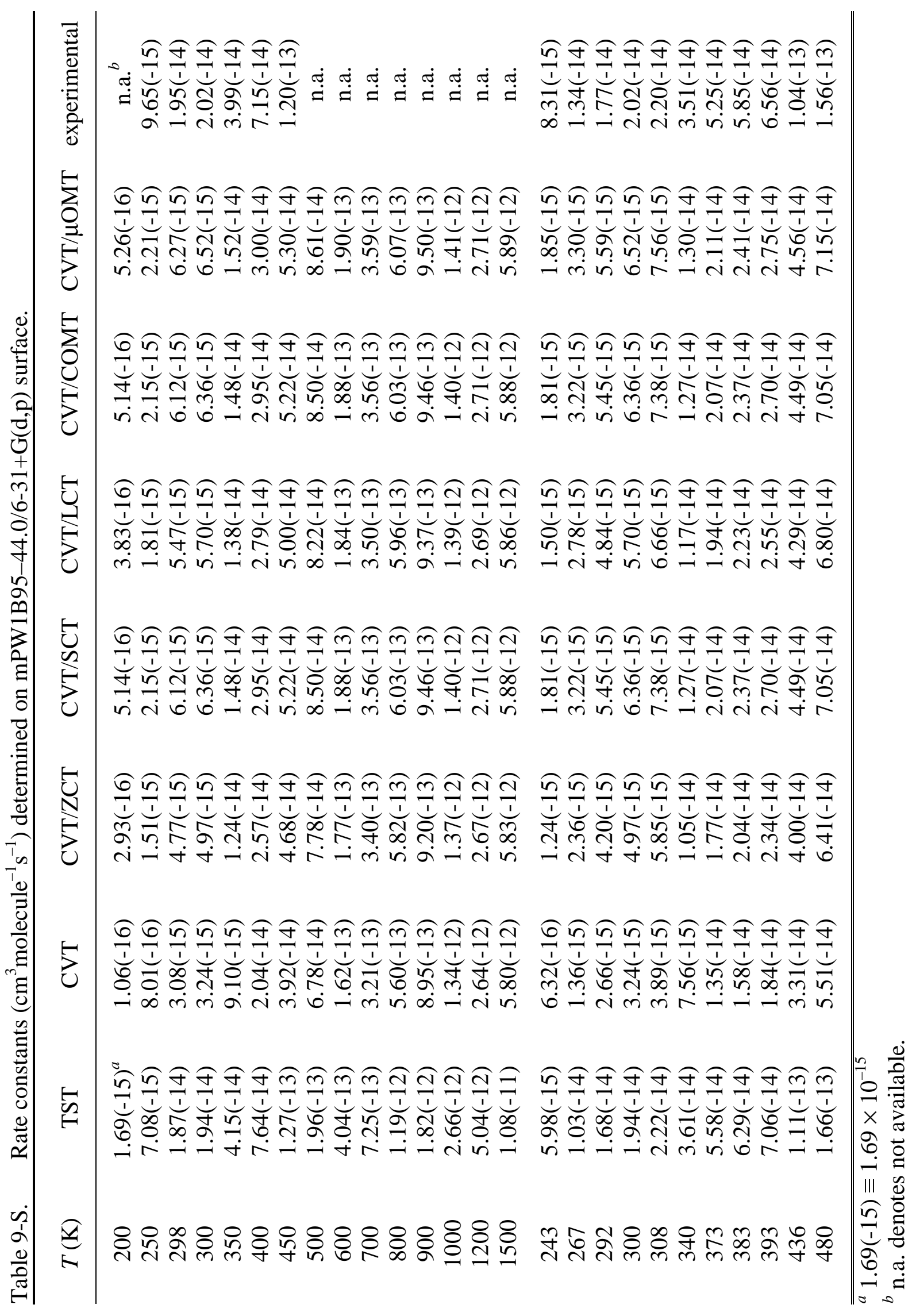


Table 10-S. Rate constants $\left(\mathrm{cm}^{3}\right.$ molecule $\left.\mathrm{s}^{-1}\right)$ determined on mPW1PW91-42.8/6-31+G(d,p) surface with all frequencies scaled by a factor of 0.9515 .

\begin{tabular}{|c|c|c|c|c|}
\hline$T(\mathrm{~K})$ & TST & CVT & $\mathrm{CVT} / \mathrm{ZCT}$ & CVT/SCT \\
\hline 200 & $1.52(-16)^{a}$ & $1.13(-17)$ & $3.81(-17)$ & $8.15(-17)$ \\
\hline 250 & $1.06(-15)$ & $1.36(-16)$ & $2.91(-16)$ & $4.77(-16)$ \\
\hline 298 & $3.88(-15)$ & $7.06(-16)$ & $1.20(-15)$ & $1.70(-15)$ \\
\hline 300 & $4.06(-15)$ & $7.49(-16)$ & $1.26(-15)$ & $1.79(-15)$ \\
\hline 350 & $1.11(-14)$ & $2.63(-15)$ & $3.84(-15)$ & $4.98(-15)$ \\
\hline 400 & $2.47(-14)$ & $6.98(-15)$ & $9.30(-15)$ & $1.13(-14)$ \\
\hline 450 & $4.75(-14)$ & $1.53(-14)$ & $1.92(-14)$ & $2.24(-14)$ \\
\hline 500 & $8.26(-14)$ & $2.95(-14)$ & $3.52(-14)$ & $4.00(-14)$ \\
\hline 600 & $2.03(-13)$ & $8.29(-14)$ & $9.33(-14)$ & $1.02(-13)$ \\
\hline 700 & $4.15(-13)$ & $1.84(-13)$ & $2.00(-13)$ & $2.14(-13)$ \\
\hline 800 & $7.49(-13)$ & $3.50(-13)$ & $3.72(-13)$ & $3.92(-13)$ \\
\hline 900 & $1.24(-12)$ & $5.99(-13)$ & $6.28(-13)$ & $6.54(-13)$ \\
\hline 1000 & $1.92(-12)$ & $9.49(-13)$ & $9.85(-13)$ & $1.02(-12)$ \\
\hline 1200 & $3.98(-12)$ & $2.02(-12)$ & $2.07(-12)$ & $2.12(-12)$ \\
\hline 1500 & $9.33(-12)$ & $4.83(-12)$ & $4.90(-12)$ & $4.97(-12)$ \\
\hline 243 & $8.42(-16)$ & $1.02(-16)$ & $2.28(-16)$ & $3.85(-16)$ \\
\hline 267 & $1.76(-15)$ & $2.59(-16)$ & $5.04(-16)$ & $7.79(-16)$ \\
\hline 292 & $3.37(-15)$ & $5.91(-16)$ & $1.03(-15)$ & $1.48(-15)$ \\
\hline 300 & $4.06(-15)$ & $7.49(-16)$ & $1.26(-15)$ & $1.79(-15)$ \\
\hline 308 & $4.86(-15)$ & $9.38(-16)$ & $1.54(-15)$ & $2.14(-15)$ \\
\hline 340 & $9.29(-15)$ & $2.10(-15)$ & $3.14(-15)$ & $4.13(-15)$ \\
\hline 373 & $1.64(-14)$ & $4.24(-15)$ & $5.91(-15)$ & $7.42(-15)$ \\
\hline 383 & $1.92(-14)$ & $5.14(-15)$ & $7.03(-15)$ & $8.73(-15)$ \\
\hline 393 & $2.23(-14)$ & $6.17(-15)$ & $8.31(-15)$ & $1.02(-14)$ \\
\hline 436 & $4.00(-14)$ & $1.25(-14)$ & $1.59(-14)$ & $1.88(-14)$ \\
\hline 480 & $6.69(-14)$ & $2.30(-14)$ & $2.79(-14)$ & $3.21(-14)$ \\
\hline
\end{tabular}

${ }^{a} 1.52(-16) \equiv 1.52 \times 10^{-16}$ 
Table 11-S. Rate constants $\left(\mathrm{cm}^{3}\right.$ molecule $\left.\mathrm{s}^{-1} \mathrm{~s}^{-1}\right)$ determined on B1B95-42.0/6-31+G(d,p) surface with all frequencies scaled by a factor of 0.9561 .

\begin{tabular}{|c|c|c|c|c|}
\hline$T(\mathrm{~K})$ & TST & CVT & CVT/ZCT & CVT/SCT \\
\hline 200 & $6.33(-16)^{a}$ & $4.70(-17)$ & $1.18(-16)$ & $1.94(-16)$ \\
\hline 250 & $3.33(-15)$ & $4.29(-16)$ & $7.62(-16)$ & $1.04(-15)$ \\
\hline 298 & $1.02(-14)$ & $1.87(-15)$ & $2.77(-15)$ & $3.45(-15)$ \\
\hline 300 & $1.06(-14)$ & $1.97(-15)$ & $2.90(-15)$ & $3.60(-15)$ \\
\hline 350 & $2.54(-14)$ & $6.06(-15)$ & $8.01(-15)$ & $9.38(-15)$ \\
\hline 400 & $5.08(-14)$ & $1.46(-14)$ & $1.79(-14)$ & $2.02(-14)$ \\
\hline 450 & $9.02(-14)$ & $2.96(-14)$ & $3.47(-14)$ & $3.81(-14)$ \\
\hline 500 & $1.47(-13)$ & $5.34(-14)$ & $6.05(-14)$ & $6.53(-14)$ \\
\hline 600 & $3.29(-13)$ & $1.37(-13)$ & $1.48(-13)$ & $1.56(-13)$ \\
\hline 700 & $6.26(-13)$ & $2.85(-13)$ & $3.00(-13)$ & $3.12(-13)$ \\
\hline 800 & $1.07(-12)$ & $5.16(-13)$ & $5.34(-13)$ & $5.51(-13)$ \\
\hline 900 & $1.70(-12)$ & $8.50(-13)$ & $8.72(-13)$ & $8.92(-13)$ \\
\hline 1000 & $2.55(-12)$ & $1.31(-12)$ & $1.33(-12)$ & $1.36(-12)$ \\
\hline 1200 & $5.04(-12)$ & $2.66(-12)$ & $2.69(-12)$ & $2.73(-12)$ \\
\hline 1500 & $1.13(-11)$ & $6.08(-12)$ & $6.10(-12)$ & $6.15(-12)$ \\
\hline 243 & $2.74(-15)$ & $3.32(-16)$ & $6.10(-16)$ & $8.50(-16)$ \\
\hline 267 & $5.14(-15)$ & $7.63(-16)$ & $1.26(-15)$ & $1.65(-15)$ \\
\hline 292 & $9.00(-15)$ & $1.59(-15)$ & $2.40(-15)$ & $3.02(-15)$ \\
\hline 300 & $1.06(-14)$ & $1.97(-15)$ & $2.90(-15)$ & $3.60(-15)$ \\
\hline 308 & $1.24(-14)$ & $2.41(-15)$ & $3.47(-15)$ & $4.26(-15)$ \\
\hline 340 & $2.17(-14)$ & $4.95(-15)$ & $6.66(-15)$ & $7.88(-15)$ \\
\hline 373 & $3.55(-14)$ & $9.30(-15)$ & $1.18(-14)$ & $1.36(-14)$ \\
\hline 383 & $4.07(-14)$ & $1.10(-14)$ & $1.39(-14)$ & $1.58(-14)$ \\
\hline 393 & $4.65(-14)$ & $1.30(-14)$ & $1.62(-14)$ & $1.83(-14)$ \\
\hline 436 & $7.76(-14)$ & $2.46(-14)$ & $2.92(-14)$ & $3.23(-14)$ \\
\hline 480 & $1.22(-13)$ & $4.27(-14)$ & $4.89(-14)$ & $5.32(-14)$ \\
\hline
\end{tabular}

${ }^{a} 6.33(-16) \equiv 6.33 \times 10^{-16}$ 
Table 12-S. Rate constants $\left(\mathrm{cm}^{3}\right.$ molecule $\left.\mathrm{s}^{-1}\right)$ determined on mPW1B95-44.0/6-31+G(d,p) surface with all frequencies scaled by a factor of 0.9537 .

\begin{tabular}{|c|c|c|c|c|}
\hline$T(\mathrm{~K})$ & TST & CVT & $\mathrm{CVT} / \mathrm{ZCT}$ & CVT/SCT \\
\hline 200 & $1.50(-15)^{a}$ & $1.15(-16)$ & $3.22(-16)$ & $5.87(-16)$ \\
\hline 250 & $6.63(-15)$ & $8.79(-16)$ & $1.67(-15)$ & $2.45(-15)$ \\
\hline 298 & $1.81(-14)$ & $3.41(-15)$ & $5.30(-15)$ & $6.94(-15)$ \\
\hline 300 & $1.88(-14)$ & $3.57(-15)$ & $5.53(-15)$ & $7.21(-15)$ \\
\hline 350 & $4.15(-14)$ & $1.01(-14)$ & $1.39(-14)$ & $1.68(-14)$ \\
\hline 400 & $7.82(-14)$ & $2.28(-14)$ & $2.89(-14)$ & $3.35(-14)$ \\
\hline 450 & $1.32(-13)$ & $4.42(-14)$ & $5.29(-14)$ & $5.95(-14)$ \\
\hline 500 & $2.08(-13)$ & $7.67(-14)$ & $8.84(-14)$ & $9.72(-14)$ \\
\hline 600 & $4.39(-13)$ & $1.85(-13)$ & $2.03(-13)$ & $2.17(-13)$ \\
\hline 700 & $8.04(-13)$ & $3.69(-13)$ & $3.93(-13)$ & $4.13(-13)$ \\
\hline 800 & $1.34(-12)$ & $6.49(-13)$ & $6.77(-13)$ & $7.03(-13)$ \\
\hline 900 & $2.07(-12)$ & $1.04(-12)$ & $1.08(-12)$ & $1.11(-12)$ \\
\hline 1000 & $3.05(-12)$ & $1.57(-12)$ & $1.61(-12)$ & $1.65(-12)$ \\
\hline 1200 & $5.86(-12)$ & $3.11(-12)$ & $3.15(-12)$ & $3.21(-12)$ \\
\hline 1500 & $1.27(-11)$ & $6.89(-12)$ & $6.94(-12)$ & $7.02(-12)$ \\
\hline 243 & $5.57(-15)$ & $6.92(-16)$ & $1.37(-15)$ & $2.05(-15)$ \\
\hline 267 & $9.81(-15)$ & $1.49(-15)$ & $2.61(-15)$ & $3.65(-15)$ \\
\hline 292 & $1.62(-14)$ & $2.94(-15)$ & $4.67(-15)$ & $6.18(-15)$ \\
\hline 300 & $1.88(-14)$ & $3.57(-15)$ & $5.53(-15)$ & $7.21(-15)$ \\
\hline 308 & $2.16(-14)$ & $4.31(-15)$ & $6.51(-15)$ & $8.37(-15)$ \\
\hline 340 & $3.60(-14)$ & $8.40(-15)$ & $1.17(-14)$ & $1.44(-14)$ \\
\hline 373 & $5.64(-14)$ & $1.51(-14)$ & $1.98(-14)$ & $2.35(-14)$ \\
\hline 383 & $6.39(-14)$ & $1.77(-14)$ & $2.29(-14)$ & $2.69(-14)$ \\
\hline 393 & $7.21(-14)$ & $2.06(-14)$ & $2.63(-14)$ & $3.07(-14)$ \\
\hline 436 & $1.15(-13)$ & $3.72(-14)$ & $4.51(-14)$ & $5.12(-14)$ \\
\hline 480 & $1.75(-13)$ & $6.22(-14)$ & $7.27(-14)$ & $8.06(-14)$ \\
\hline
\end{tabular}

${ }^{a} 1.50(-15) \equiv 1.50 \times 10^{-15}$ 
Table 13-S. The parameters obtained form fitting the $\log k^{\mathrm{CVT} / \mu \mathrm{OMT}}\left(\mathrm{cm}^{3}\right.$ molecule $\left.\mathrm{s}^{-1} \mathrm{~s}^{-1}\right) \mathrm{vs}$. the HF exchange contribution $(X)$ to the quadratic equation:

\begin{tabular}{|c|c|c|c|c|}
\hline Functional & $T(\mathrm{~K})$ & $a \times 10^{3}$ & $b$ & $c$ \\
\hline \multirow[t]{11}{*}{ mPW1PW91-X } & 243 & 1.0488 & -0.2624 & -6.1677 \\
\hline & 267 & 0.8776 & -0.2365 & -6.6546 \\
\hline & 292 & 0.7831 & -0.2177 & -7.0045 \\
\hline & 300 & 0.6917 & -0.2076 & -7.1916 \\
\hline & 308 & 0.7076 & -0.2055 & -7.2296 \\
\hline & 340 & 0.5911 & -0.1850 & -7.6101 \\
\hline & 373 & 0.5411 & -0.1709 & -7.8678 \\
\hline & 383 & 0.4262 & -0.1592 & -8.0866 \\
\hline & 393 & 0.5137 & -0.1634 & -8.0010 \\
\hline & 436 & 0.4184 & -0.1456 & -8.3221 \\
\hline & 480 & 0.3908 & -0.1346 & -8.5112 \\
\hline \multirow[t]{11}{*}{ В1B95- $X$} & 243 & 1.2842 & -0.2781 & -5.7073 \\
\hline & 267 & 1.0644 & -0.2484 & -6.2764 \\
\hline & 292 & 0.8116 & -0.2177 & -6.8614 \\
\hline & 300 & 0.8306 & -0.2156 & -6.9031 \\
\hline & 308 & 0.8791 & -0.2161 & -6.8971 \\
\hline & 340 & 0.6895 & -0.1899 & -7.3936 \\
\hline & 373 & 0.6314 & -0.1752 & -7.6736 \\
\hline & 383 & 0.5052 & -0.1629 & -7.9025 \\
\hline & 393 & 0.4541 & -0.1563 & -8.0275 \\
\hline & 436 & 0.6171 & -0.1586 & -7.9707 \\
\hline & 480 & 0.3707 & -0.1308 & -8.4872 \\
\hline \multirow[t]{11}{*}{ mPW1B95- $X$} & 243 & 1.9149 & -0.3404 & -3.4630 \\
\hline & 267 & 1.8427 & -0.3214 & -3.9071 \\
\hline & 292 & 1.8183 & -0.3077 & -4.2342 \\
\hline & 300 & 1.6926 & -0.2941 & -4.5239 \\
\hline & 308 & 1.5841 & -0.2819 & -4.7865 \\
\hline & 340 & 1.3479 & -0.2507 & -5.4632 \\
\hline & 373 & 1.1946 & -0.2279 & -5.9618 \\
\hline & 383 & 1.1655 & -0.2229 & -6.0658 \\
\hline & 393 & 1.1025 & -0.2146 & -6.2513 \\
\hline & 436 & 1.0139 & -0.1974 & -6.6193 \\
\hline & 480 & 0.9606 & -0.1840 & -6.9091 \\
\hline
\end{tabular}




Method: mPWPW91-33.0/6-31+G $(\mathrm{d}, \mathrm{p})$
$\begin{array}{lrrr}\mathrm{CH}_{3} \mathrm{~F}: \\ \mathrm{F} 1 & 0.000000 & 0.000000 & 1.211373 \\ \mathrm{C} 2 & 0.000000 & 0.000001 & -1.398869 \\ \mathrm{H} 3 & 0.000000 & 1.949804 & -2.059785 \\ \mathrm{H} 4 & -1.688579 & -0.974902 & -2.059784 \\ \mathrm{H} 5 & 1.688579 & -0.974902 & -2.059784\end{array}$

$\begin{array}{lrl}\text { Energy: } & -139.71221719860 \mathrm{a} . \mathrm{u} . & \\ \text { Frequencies: } & 3200.20,3200.19,3100.37,1518.16,1518.16,1508.62, \\ & 1211.96,1211.93,1101.30\end{array}$

$\mathrm{OH}$ :

$\begin{array}{llll}06 & 0.000000 & 0.000000 & -0.108727\end{array}$

H7 $0.000000 \quad 0.000000 \quad 1.725583$

$\begin{array}{lrrr}\begin{array}{l}\text { Energy: } \\ \text { Frequency: }\end{array} & -75.715007927829 \mathrm{a} . \mathrm{u} . \\ & 3828.78 & \\ \mathrm{CH}_{2} \mathrm{~F}: & & \\ \mathrm{F} 1 & -0.027965 & -1.124395 & 0.000000 \\ \mathrm{C} 2 & -0.023511 & 1.402332 & 0.000000 \\ \mathrm{H} 4 & 0.403552 & 2.249265 & 1.806160 \\ \mathrm{H} 5 & 0.403552 & 2.249265 & -1.806160\end{array}$

Energy: $\quad-139.04158440505$ a.u.

Frequencies: $\quad 3370.09,3208.26,1491.10,1216.43,1189.48,570.19$

$\mathrm{H}_{2} \mathrm{O}$ :

H3 $\quad 0.000000 \quad 1.443851-0.966660$

$06 \quad 0.000000 \quad 0.000000 \quad 0.121817$

H7 $\quad 0.000000 \quad-1.443851-0.966660$

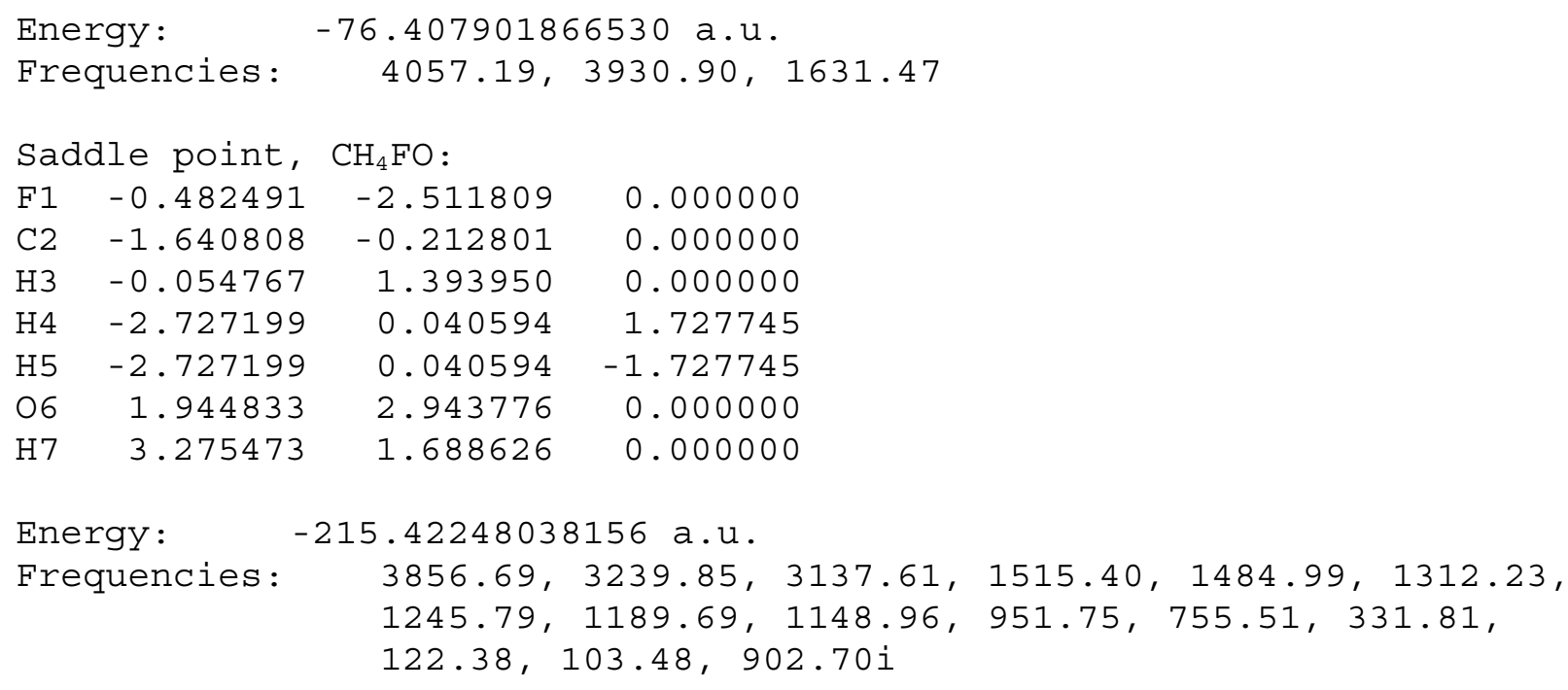




Method: mPWPW91-35.7/6-31+G $(\mathrm{d}, \mathrm{p})$
$\begin{array}{lrrr}\mathrm{CH}_{3} \mathrm{~F}: \\ \mathrm{F} 1 & 0.000000 & 0.000000 & 1.209869 \\ \mathrm{C} 2 & 0.000000 & 0.000001 & -1.397010 \\ \mathrm{H} 3 & 0.000000 & 1.948245 & -2.057718 \\ \mathrm{H} 4 & -1.687229 & -0.974123 & -2.057718 \\ \mathrm{H} 5 & 1.687229 & -0.974123 & -2.057718\end{array}$

$\mathrm{OH}$ :

$06 \quad 0.000000 \quad 0.000000 \quad-0.108590$

H7 $0.000000 \quad 0.000000 \quad 1.723405$

$\begin{array}{lrrr}\text { Energy: } & -75.714205048657 \mathrm{a} . \mathrm{u} . \\ \text { Frequency: } & 3845.64 & \\ & & & \\ \mathrm{CH}_{2} \mathrm{~F}: & & & \\ \mathrm{F} 1 & -0.027513 & -1.123223 & 0.000000 \\ \mathrm{C} 2 & -0.024261 & 1.400805 & 0.000000 \\ \mathrm{H} 4 & 0.403757 & 2.247305 & 1.804461 \\ \mathrm{H} 5 & 0.403757 & 2.247305 & -1.804461\end{array}$

Energy: $\quad-139.04002737479$ a.u.

Frequencies: $\quad 3379.22,3217.27,1496.35,1222.05,1193.75,575.71$

$\mathrm{H}_{2} \mathrm{O}$ :

H3 $0.000000 \quad 1.442835 \quad-0.964772$

$06 \quad 0.000000 \quad 0.000000 \quad 0.121579$

H7 $\quad 0.000000 \quad-1.442835 \quad-0.964772$

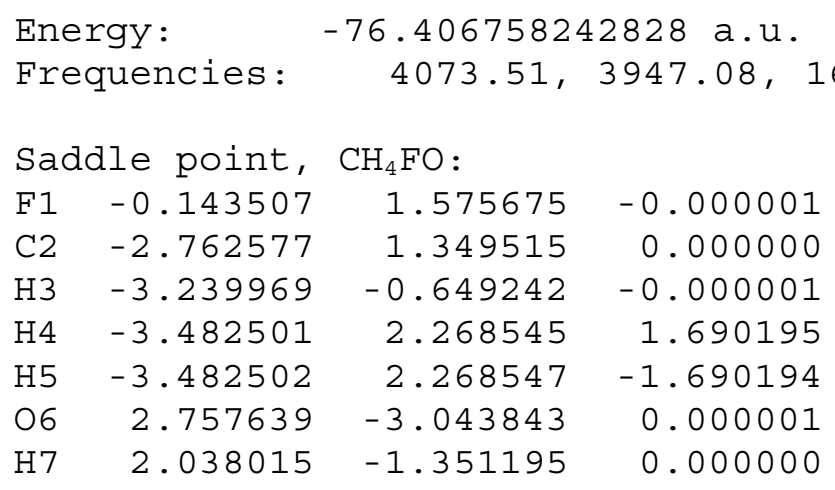

Energy: $\quad-215.43142356627$ a.u.

Frequencies: $\quad 3874.39,3250.74,3147.37,1520.29,1489.43,1312.31$, $1246.52,1192.83,1153.82,896.40,754.87,337.40$, $123.79,105.12,1041.55 i$ 


Method: mPWPW91-42.8/6-31+G $(\mathrm{d}, \mathrm{p})$
$\begin{array}{lrrr}\mathrm{CH}_{3} \mathrm{~F}: \\ \mathrm{F} 1 & 0.000000 & 0.000000 & 1.206037 \\ \mathrm{C} 2 & 0.000000 & 0.000000 & -1.392281 \\ \mathrm{H} 3 & 0.000000 & 1.944245 & -2.052408 \\ \mathrm{H} 4 & -1.683767 & -0.972122 & -2.052408 \\ \mathrm{H} 5 & 1.683767 & -0.972122 & -2.052408\end{array}$

$\begin{array}{lrl}\text { Energy: } & -139.70728712322 \text { a.u. } & \\ \text { Frequencies: } & 3233.93,3233.92,3132.51,1536.66,1536.66,1527.91, \\ & 1228.53,1228.50,1124.82\end{array}$

$\mathrm{OH}$ :

$06 \quad 0.000000 \quad 0.000000 \quad-0.108238$

H7 $0.000000 \quad 0.000000 \quad 1.717821$

$\begin{array}{lrrr}\text { Energy: } & -75.712169001305 \mathrm{a} . \mathrm{u} . \\ \text { Frequency: } & 3889.12 & \\ & & & \\ \mathrm{CH}_{2} \mathrm{~F}: & & & \\ \mathrm{F} 1 & -0.026293 & -1.120241 & 0.000000 \\ \mathrm{C} 2 & -0.026293 & 1.396935 & 0.000000 \\ \mathrm{H} 4 & 0.404351 & 2.242241 & 1.800078 \\ \mathrm{H} 5 & 0.404351 & 2.242241 & -1.800078\end{array}$

Energy: $\quad-139.03610962870$ a.u.

Frequencies: $\quad 3402.75,3240.50,1509.94,1236.41,1204.83,590.37$

$\mathrm{H}_{2} \mathrm{O}$ :

H3 $0.000000 \quad 1.440196 \quad-0.959967$

$06 \quad 0.000000 \quad 0.000000 \quad 0.120973$

H7 $\quad 0.000000 \quad-1.440196 \quad-0.959967$

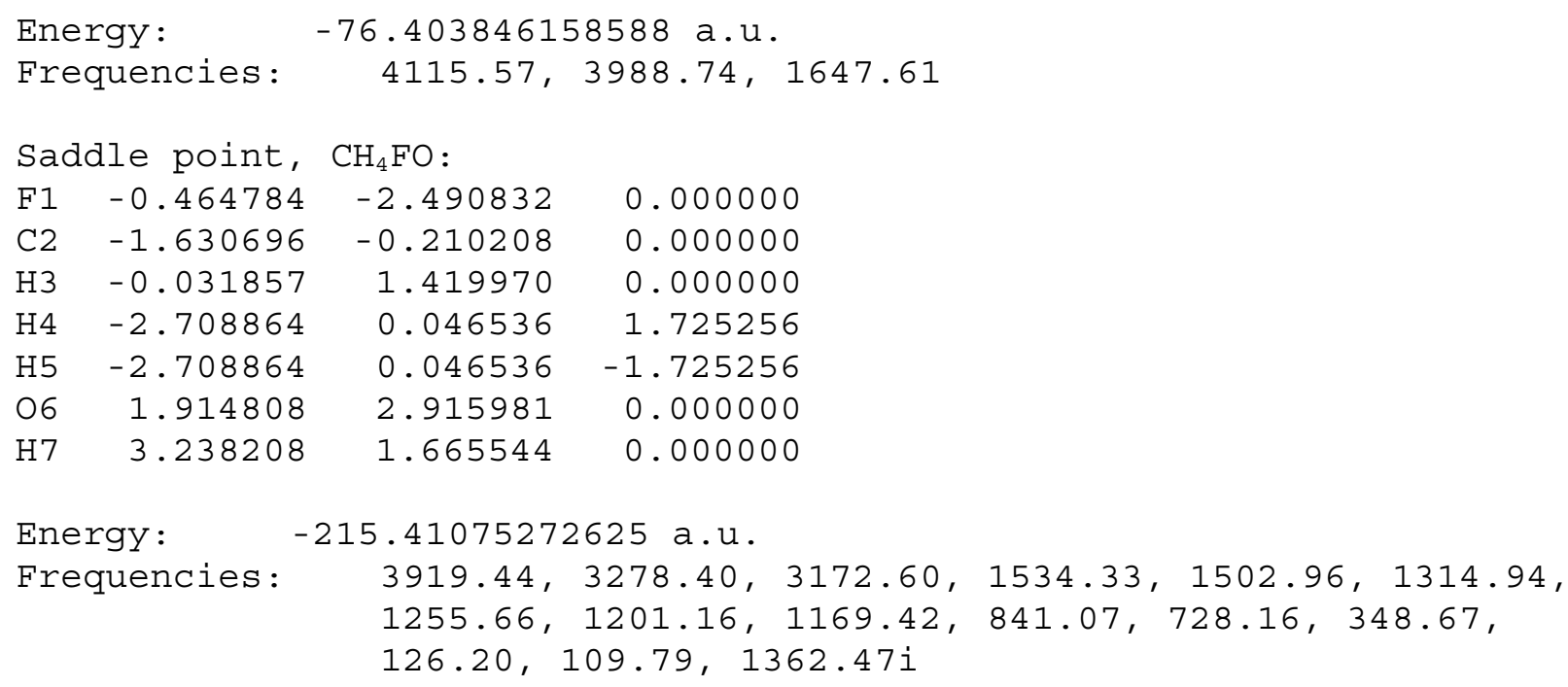


Method: $B 1 B 95-34 \cdot 3 / 6-31+G(d, p)$

$\begin{array}{lrrr}\mathrm{CH}_{3} \mathrm{~F}: & & & \\ \mathrm{F} 1 & 0.000000 & 0.000001 & 1.210827 \\ \mathrm{C} 2 & 0.000000 & 0.000000 & -1.398242 \\ \mathrm{H} 3 & 0.000000 & 1.948817 & -2.058842 \\ \mathrm{H} 4 & -1.687720 & -0.974415 & -2.058849 \\ \mathrm{H} 5 & 1.687720 & -0.974415 & -2.058849\end{array}$

Energy: $\quad-139.69701134986$ a.u.

Frequencies: $\quad 3193.92,3193.83,3095.13,1512.10,1512.04,1501.82$, $1206.76,1206.64,1102.05$

$\mathrm{OH}$ :

$06 \quad 0.000000 \quad 0.000000 \quad-0.108855$

H7 $0.000000 \quad 0.000000 \quad 1.727602$

$\begin{array}{lrrr}\begin{array}{l}\text { Energy: } \\ \text { Frequency: }\end{array} & -75.707211479881 \mathrm{a} . \mathrm{u} . \\ & 3804.68 & \\ \mathrm{CH}_{2} \mathrm{~F}: & & \\ \mathrm{F} 1 & -0.023957 & -1.123132 & 0.000000 \\ \mathrm{C} 2 & -0.030554 & 1.401099 & 0.000000 \\ \mathrm{H} 4 & 0.407707 & 2.244698 & 1.805138 \\ \mathrm{H} 5 & 0.407707 & 2.244698 & -1.805138\end{array}$

Energy: $\quad-139.02791325645$ a.u.

Frequencies: $\quad 3357.11,3194.47,1485.27,1218.25,1187.40,587.27$

$\mathrm{H}_{2} \mathrm{O}$ :

H3 $0.000000 \quad 1.443892 \quad-0.967599$

$06 \quad 0.000000 \quad 0.000000 \quad 0.121935$

H7 $\quad 0.000000 \quad-1.443892 \quad-0.967599$

\begin{tabular}{lrrr} 
Energy: & $-76.398803100494 \mathrm{a} . \mathrm{u}$. \\
Frequencies: & $4042.53,3916.53,1$ \\
\multicolumn{4}{l}{ Saddle point, } \\
$\begin{array}{l}\text { F1 } \\
\text { F }\end{array}$-0.181532 & 1.571991 & 0.000000 \\
$\mathrm{C} 2$ & -2.804355 & 1.379202 & 0.000000 \\
H3 & -3.308082 & -0.613571 & 0.000000 \\
H4 & -3.513292 & 2.307515 & 1.690538 \\
H5 & -3.513292 & 2.307515 & -1.690538 \\
O6 & 2.839179 & -3.066811 & 0.000000 \\
H7 & 2.087870 & -1.384296 & 0.000000
\end{tabular}

Energy: $\quad-215.40977495308$ a.u.

Frequencies: $\quad 3838.78,3232.20,3130.29,1508.39,1477.33,1309.27$, $1236.24,1184.97,1150.60,978.71,751.17,328.27$, $120.62,104.99,832.91 i$ 


$$
\begin{aligned}
& \text { Method: B1B95-37.0/6-31+G }(\mathrm{d}, \mathrm{p}) \\
& \begin{array}{lrrr}
\mathrm{CH}_{3} \mathrm{~F}: \\
\mathrm{F} 1 & 0.000000 & 0.000001 & 1.209299 \\
\mathrm{C} 2 & 0.000000 & 0.000000 & -1.396358 \\
\mathrm{H} 3 & 0.000000 & 1.947222 & -2.056718 \\
\mathrm{H} 4 & -1.686338 & -0.973618 & -2.056724 \\
\mathrm{H} 5 & 1.686338 & -0.973618 & -2.056724
\end{array}
\end{aligned}
$$


Method: $B 1 B 95-42.0 / 6-31+G(d, p)$

$\begin{array}{lrrr}\mathrm{CH}_{3} \mathrm{~F}: & & & \\ \mathrm{F} 1 & 0.000000 & 0.000001 & 1.206538 \\ \mathrm{C} 2 & 0.000000 & 0.000000 & -1.392960 \\ \mathrm{H} 3 & 0.000000 & 1.944327 & -2.052854 \\ \mathrm{H} 4 & -1.683831 & -0.972171 & -2.052861 \\ \mathrm{H} 5 & 1.683831 & -0.972171 & -2.052861\end{array}$

$\begin{array}{lrl}\text { Energy: } & -139.69286419816 \text { a.u. } \\ \text { Frequencies: } & 3221.45,3221.36,3121.43,1526.84,1526.77,1517.15, \\ & 1219.91,1219.79,1120.98\end{array}$

$\mathrm{OH}$ :

$06 \quad 0.000000 \quad 0.000000 \quad-0.108461$

H7 $0.000000 \quad 0.000000 \quad 1.721347$

$\begin{array}{lrrr}\begin{array}{l}\text { Energy: } \\ \text { Frequency: }\end{array} & -75.704692742412 \mathrm{a} . \mathrm{u} . \\ & 3852.82 & \\ \mathrm{CH}_{2} \mathrm{~F}: & & \\ \mathrm{F} 1 & -0.022754 & -1.119752 & 0.000000 \\ \mathrm{C} 2 & -0.032537 & 1.396694 & 0.000000 \\ \mathrm{H} 4 & 0.408176 & 2.239069 & 1.800229 \\ \mathrm{H} 5 & 0.408176 & 2.239069 & -1.800229\end{array}$

Energy: $\quad-139.02324078436$ a.u.

Frequencies: $\quad 3383.76,3220.82,1500.33,1234.24,1199.58,603.18$

$\mathrm{H}_{2} \mathrm{O}$ :

H3 $0.000000 \quad 1.441009-0.962157$

$06 \quad 0.000000 \quad 0.000000 \quad 0.121249$

H7 $0.000000 \quad-1.441009-0.962157$

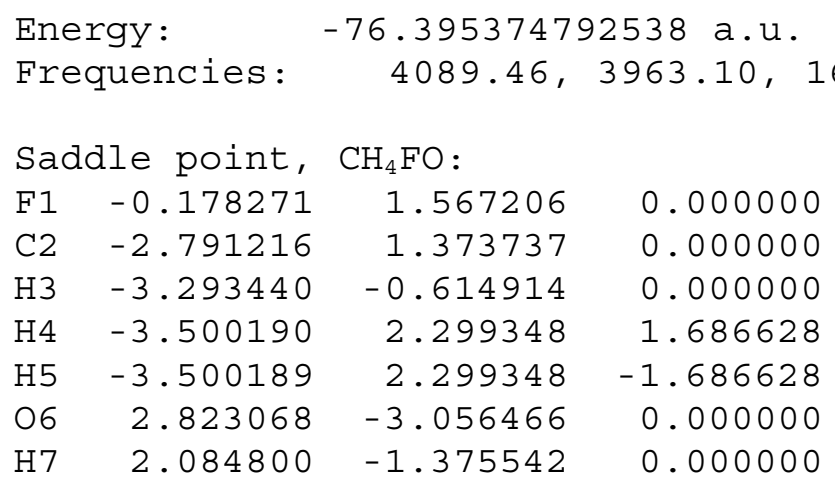

Energy: $\quad-215.40332315449$ a.u.

Frequencies: $\quad 3889.18,3263.33,3158.55,1522.99,1491.15,1311.01$, $1244.48,1194.08,1165.96,852.96,738.81,342.08$, $123.37,109.64,1206.78 i$ 


Method: mPW1B95-38.0/6-31+G $(\mathrm{d}, \mathrm{p})$
$\begin{array}{lrrr}\mathrm{CH}_{3} \mathrm{~F}: \\ \mathrm{F} 1 & 0.000000 & 0.000000 & 1.208362 \\ \mathrm{C} 2 & 0.000000 & 0.000000 & -1.395259 \\ \mathrm{H} 3 & 0.000000 & 1.946479 & -2.055191 \\ \mathrm{H} 4 & -1.685695 & -0.973246 & -2.055197 \\ \mathrm{H} 5 & 1.685695 & -0.973246 & -2.055197\end{array}$
Energy: $\quad-139.69352202609$ a.u.
Frequencies: $\quad 3208.16,3208.08,3108.87,1518.46,1518.40,1508.43$, $1212.70,1212.58,1112.22$

$\mathrm{OH}$ :

$06 \quad 0.000000 \quad 0.000000 \quad-0.108668$

H7 $0.000000 \quad 0.000000 \quad 1.724637$

$\begin{array}{lrrr}\text { Energy: } & -75.704524039836 \mathrm{a} . \mathrm{u} . \\ \text { Frequency: } & 3827.55 & \\ & & & \\ \mathrm{CH}_{2} \mathrm{~F}: & & & \\ \mathrm{F} 1 & -0.024073 & -1.121027 & 0.000000 \\ \mathrm{C} 2 & -0.030233 & 1.398358 & 0.000000 \\ \mathrm{H} 4 & 0.406893 & 2.241179 & 1.803053 \\ \mathrm{H} 5 & 0.406893 & 2.241179 & -1.803053\end{array}$

Energy: $\quad-139.02423702588$ a.u.

Frequencies: $\quad 3371.65,3208.58,1491.87,1227.52,1192.86,590.52$

$\mathrm{H}_{2} \mathrm{O}$ :

H3 $0.000000 \quad 1.442999 \quad-0.964410$

$06 \quad 0.000000 \quad 0.000000 \quad 0.121533$

H7 $0.000000 \quad-1.442999 \quad-0.964410$

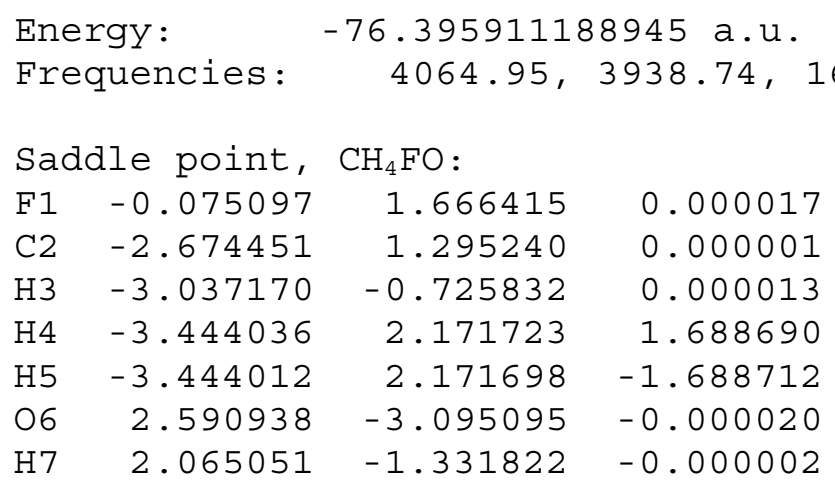

Energy: $\quad-215.40463279767 \mathrm{a} . \mathrm{u}$.

Frequencies: $\quad 3861.64,3247.71,3144.66,1515.19,1482.25,1309.84$, $1241.81,1188.82,1158.13,917.99,750.74,330.89$, $118.71,110.08,984.39 i$ 


Method: mPW1B95-41.0/6-31+G $(\mathrm{d}, \mathrm{p})$
$\begin{array}{lrrr}\mathrm{CH}_{3} \mathrm{~F}: \\ \mathrm{F} 1 & 0.000000 & 0.000001 & 1.206723 \\ \mathrm{C} 2 & 0.000000 & 0.000000 & -1.393239 \\ \mathrm{H} 3 & 0.000000 & 1.944749 & -2.052914 \\ \mathrm{H} 4 & -1.684196 & -0.972381 & -2.052920 \\ \mathrm{H} 5 & 1.684196 & -0.972381 & -2.052920\end{array}$
Energy: $\quad-139.69198132631$ a.u.
Frequencies: $\quad 3218.78,3218.69,3119.00,1524.22,1524.16,1514.41$, $1217.83,1217.70,1119.51$

$\mathrm{OH}$ :

$\begin{array}{llll}06 & 0.000000 & 0.000000 & -0.108515\end{array}$

H7 $0.000000 \quad 0.000000 \quad 1.722216$

$\begin{array}{lrrr}\begin{array}{l}\text { Energy: } \\ \text { Frequency: }\end{array} & -75.703609610281 \mathrm{a} . \mathrm{u} . \\ & 3846.22 & \\ \mathrm{CH}_{2} \mathrm{~F}: & & \\ \mathrm{F} 1 & -0.023555 & -1.119745 & 0.000000 \\ \mathrm{C} 2 & -0.031096 & 1.396687 & 0.000000 \\ \mathrm{H} 4 & 0.407148 & 2.239037 & 1.801132 \\ \mathrm{H} 5 & 0.407148 & 2.239037 & -1.801132\end{array}$

Energy: $\quad-139.02248735181 \mathrm{a} . \mathrm{u}$.

Frequencies: $\quad 3381.88,3218.71,1497.74,1233.63,1197.61,596.98$

$\mathrm{H}_{2} \mathrm{O}$ :

$\begin{array}{lllr}\mathrm{H} 3 & 0.000000 & 1.441852 & -0.962344 \\ 06 & 0.000000 & 0.000000 & 0.121273\end{array}$

H7 $\quad 0.000000-1.441852 \quad-0.962344$

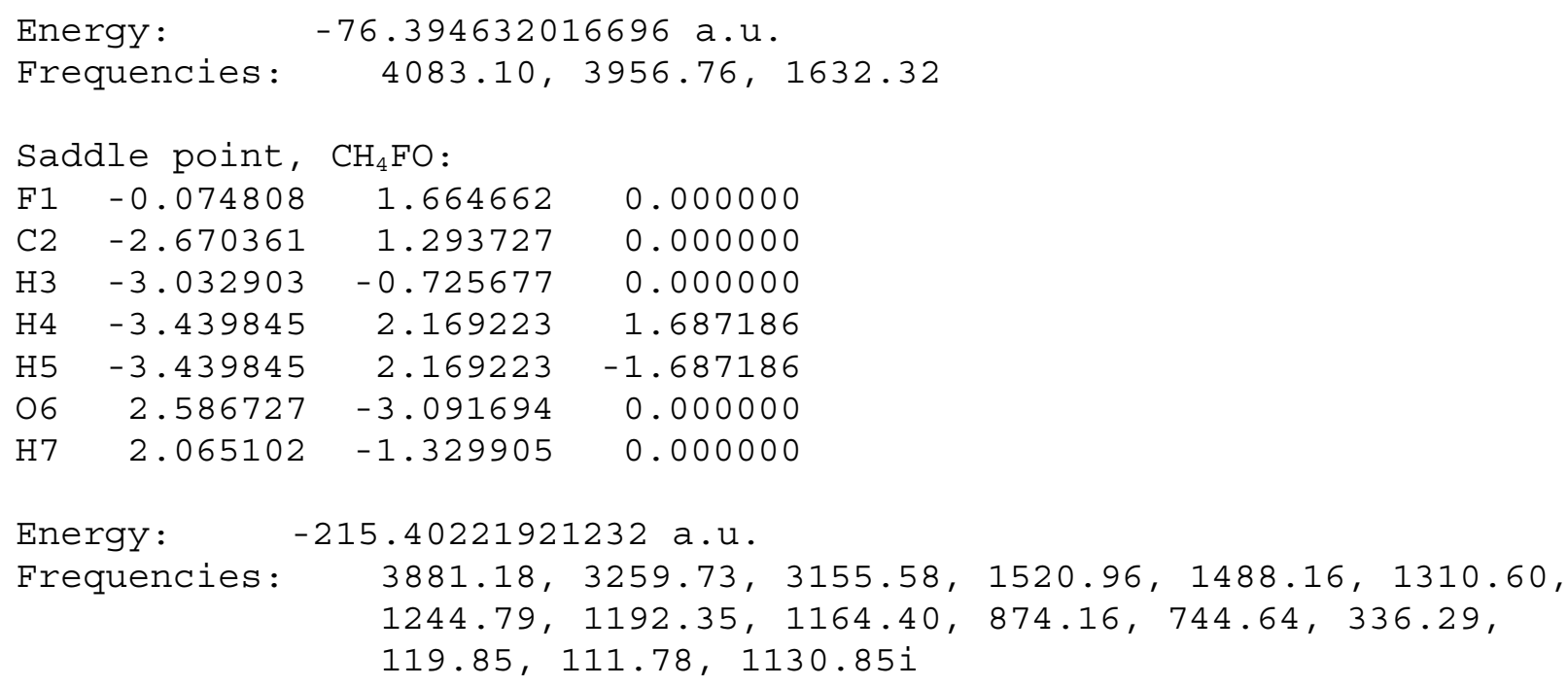




Method: mPW1B95-44.0/6-31+G $(\mathrm{d}, \mathrm{p})$
$\begin{array}{lrrr}\mathrm{CH}_{3} \mathrm{~F}: \\ \mathrm{F} 1 & 0.000000 & 0.000001 & 1.205116 \\ \mathrm{C} 2 & 0.000000 & 0.000000 & -1.391261 \\ \mathrm{H} 3 & 0.000000 & 1.943045 & -2.050668 \\ \mathrm{H} 4 & -1.682721 & -0.971529 & -2.050674 \\ \mathrm{H} 5 & 1.682721 & -0.971529 & -2.050674\end{array}$

$\begin{array}{lrl}\text { Energy: } & -139.69048572795 \mathrm{a} . \mathrm{u} . & \\ \text { Frequencies: } & 3229.27,3229.19,3129.02,1529.93,1529.87,1520.35, \\ & 1222.91,1222.78,1126.67\end{array}$

$\mathrm{OH}$ :

$06 \quad 0.000000 \quad 0.000000 \quad-0.108365$

H7 $0.000000 \quad 0.000000 \quad 1.719831$

$\begin{array}{lrrr}\begin{array}{l}\text { Energy: } \\ \text { Frequency: }\end{array} & -75.702714789611 \mathrm{a} . \mathrm{u} . \\ & 3864.66 & \\ \mathrm{CH}_{2} \mathrm{~F}: & & & \\ \mathrm{F} 1 & -0.023031 & -1.118489 & 0.000000 \\ \mathrm{C} 2 & -0.031972 & 1.395055 & 0.000000 \\ \mathrm{H} 4 & 0.407415 & 2.236917 & 1.799234 \\ \mathrm{H} 5 & 0.407415 & 2.236917 & -1.799234\end{array}$

Energy: $\quad-139.02078410844$ a.u.

Frequencies: $\quad 3391.99,3228.71,1503.56,1239.64,1202.32,603.46$

$\mathrm{H}_{2} \mathrm{O}$ :

$\begin{array}{llll}\text { H3 } & 0.000000 & 1.440713 & -0.960319\end{array}$

$06 \quad 0.000000 \quad 0.000000 \quad 0.121018$

H7 $\quad 0.000000 \quad-1.440713 \quad-0.960319$

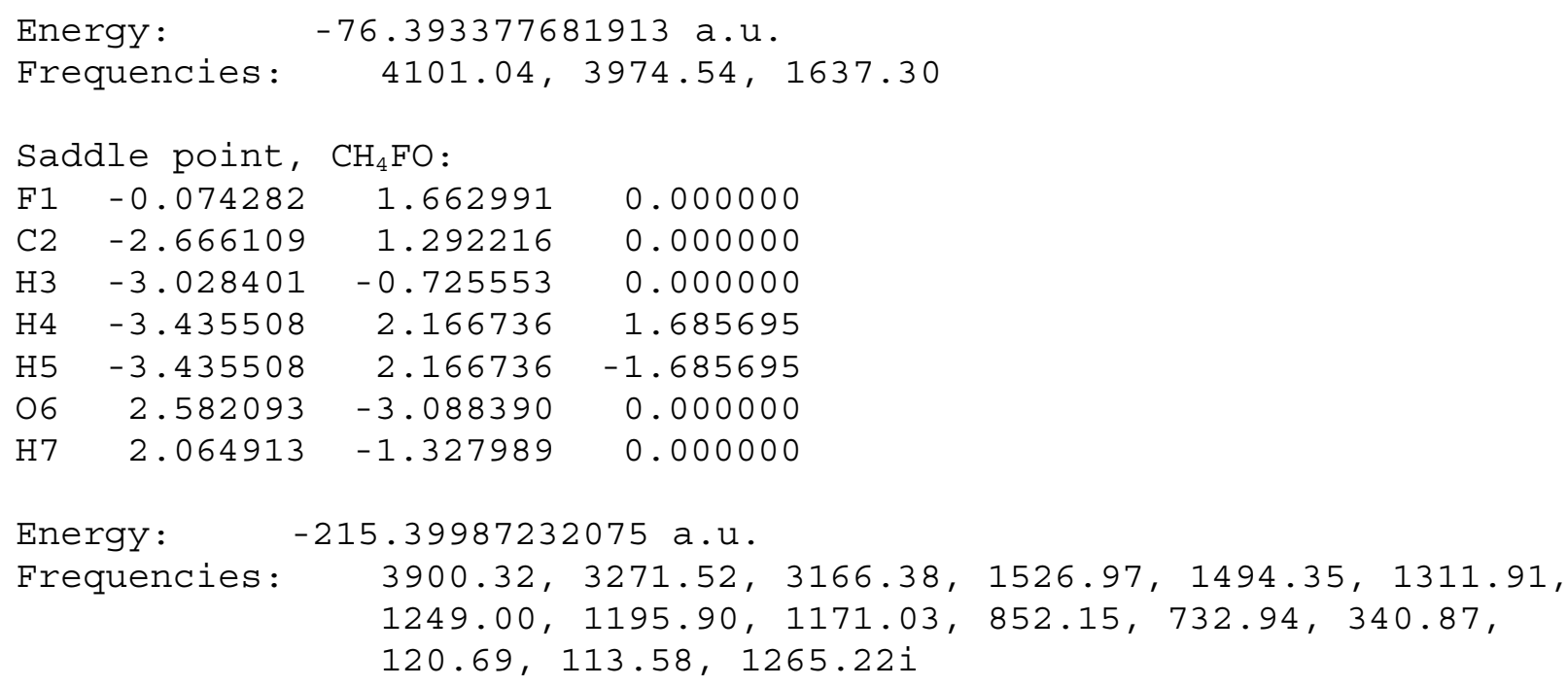

